\title{
Review Article \\ Integrating Gene Correction in the Reprogramming and Transdifferentiation Processes: A One-Step Strategy to Overcome Stem Cell-Based Gene Therapy Limitations
}

\author{
Seo-Young Lee and Sun-Ku Chung \\ Medical Research Division, Korea Institute of Oriental Medicine, 1672 Yuseong-daero, Yuseong-gu, Daejeon 34054, Republic of Korea \\ Correspondence should be addressed to Sun-Ku Chung; skchung@kiom.re.kr
}

Received 19 July 2016; Revised 9 November 2016; Accepted 16 November 2016

Academic Editor: James Adjaye

Copyright ( $\odot 2016$ S.-Y. Lee and S.-K. Chung. This is an open access article distributed under the Creative Commons Attribution License, which permits unrestricted use, distribution, and reproduction in any medium, provided the original work is properly cited.

\begin{abstract}
The recent advent of induced pluripotent stem cells (iPSCs) and gene therapy tools has raised the possibility of autologous cell therapy for rare genetic diseases. However, cellular reprogramming is inefficient in certain diseases such as ataxia telangiectasia, Fanconi anemia, LIG4 syndrome, and fibrodysplasia ossificans progressiva syndrome, owing to interference of the disease-related genes. To overcome these therapeutic limitations, it is necessary to fundamentally correct the abnormal gene during or prior to the reprogramming process. In addition, as genetic etiology of Parkinson's disease, it has been well known that induced neural stem cells (iNSCs) were progressively depleted by LRRK2 gene mutation, LRRK2 (G2019S). Thus, to maintain the induced NSCs directly derived from PD patient cells harboring LRRK2 (G2019S), it would be ideal to simultaneously treat the LRRK2 (G2019S) fibroblast during the process of TD. Therefore, simultaneous reprogramming (or TD) and gene therapy would provide the solution for therapeutic limitation caused by vulnerability of reprogramming or TD, in addition to being suitable for general application to the generation of autologous cell-therapy products for patients with genetic defects, thereby obviating the need for the arduous processes currently required.
\end{abstract}

\section{Introduction}

Since their discovery in 2006, induced pluripotent stem cells (iPSCs) have been considered to be highly useful resources for cell-replacement therapy as well as for studying human disease. Thus, iPSCs are expected to be applicable to the treatment of a broad range of diseases, including neurological disorders, hematological abnormalities, spinal cord injury, heart disease, diabetes, and arthritis $[1,2]$. Several groups have already reported the generation of a variety of iPSCs derived from patients with genetic disorders such as amyotrophic lateral sclerosis, familial dysautonomia, spinal muscular atrophy, adenosine deaminase deficiencyrelated severe combined immunodeficiency, dyskeratosis congenita, Shwachman-Bodian-Diamond syndrome, leopard syndrome, Gaucher disease type III, Duchenne muscular dystrophy, Becker muscular dystrophy, Timothy syndrome, Parkinson's disease (PD), Huntington's disease, HutchinsonGilford progeria syndrome, juvenile-onset type 1 diabetes mellitus, Down syndrome, Rett's syndrome, and LeschNyhan syndrome [3-11]. Fortunately, these disease-related iPSCs were generated without the negative influences of genetic mutations. Although disease-related genes may potentially exert adverse effects on the reprogramming process, leading to poor reprogramming efficiency and inhibitory maintenance, this is not considered a crucial concern unless the gene mutations are so severe to bring about very early embryonic lethality. Nevertheless, even for inherited genetic disorders without severe lethality in the embryonic development stage, certain disease-related genes can seriously impede the reprogramming process or impair the maintenance of iPSCs, which has been observed in cases of ataxia telangiectasia (A-T) [12], Fanconi anemia (FA) [13, 14], LIG4 syndrome [15], and fibrodysplasia ossificans progressiva (FOP) syndrome [16, 17]. Therefore, it is important to generate gene-corrected iPSCs to avoid the potential of reprogramming impairment by interference of a defective gene. To achieve this, it is necessary to genetically treat the 
iPSCs from the somatic cell phase prior to reaching the impaired iPSCs stage.

Another strategy for cell-replacement therapy is transdifferentiation (TD), also known as direct reprogramming, which is a process in which lineage-specific cell types are directly derived from somatic cell types, thereby bypassing the pluripotency stage. TD possesses several advantages such as the rapid generation of specific cell types as well as avoidance of the teratoma formation caused by the intrinsic characteristics of iPSCs. However, the TD-mediated lineagespecific cells may also be impaired by disease-related genes. A prime example of this effect is the G2019S mutant of leucine-rich repeat kinase 2 (LRRK2), which leads to nuclear disruption in induced neural stem cells (iNSCs) and has been detected in brain slices of PD patients [18]. Although NSCs can be successfully generated from iPSCs with the LRRK G2019S mutation, they are completely depleted after several passages due to abnormal interactions between LRRK2 (G2019S) and lamin B1 protein, which is anchored to the inner nuclear membrane and is involved in breaking the framework of the nuclear envelope [18]. Thus, this interaction would have a negative effect on the formation of NSCs directly derived from PD patient cells via the TD process. Therefore, along with the concurrent reprogramming and gene-correction approach, the LRRK2 (G2019S) gene would be fundamentally treated using gene-correction tools during the TD process rather than at the NSC stage in which the cells will still harbor the mutant gene.

In this review, we highlight the challenges facing current iPSC-based therapy approaches and introduce the onestep gene-correction and reprogramming approach as the solution to overcome iPSC-based gene therapy limitations. And we also propose adoption of an ideal gene therapy approach that combines the gene-correction process with the TD process, focusing on the example of the pathogenic LRRK2 mutant (G2019S), which progressively depletes neural stem cells in Parkinson's disease.

\section{Genetic Defects That Affect Reprogramming}

2.1. DNA Repair Defects Affecting the Reprogramming Process. One of the main challenges of the reprogramming process is interference owing to genome instability or apoptosis induction $[20,21]$. During this process, p53 protein, a crucial monitor of genome integrity, accumulates in response to the ectopic overexpression of reprogramming factors [22]; thus, p53 strictly regulates the reprogramming of somatic cells and can impede this process overall. Accordingly, temporary reduction in the activity of p53 can successfully enhance the reprogramming efficiency by no less than 100 times the original value [23-26], owing to the lack of concern about apoptosis and DNA damage [27]. As one of the kinases that activates $\mathrm{p} 53$ in the DNA damage response, the ataxiatelangiectasia mutated (ATM) gene also plays a pivotal role in regulating genome stability. Chromosomal instability caused by ATM mutation results in the development of $\mathrm{A}-\mathrm{T}$, a rare inherited disorder that is characterized by motor neurodegeneration, leukemia, and premature ageing [28]. The broad phenotypes associated with A-T have an additional influence on reprogramming efficiency. Consequently, the efficiency of iPSCs generated from A-T fibroblasts is extremely low, at about only $4 \%$, in A-T homozygote cells compared with normal cells $[12,29]$.

FA syndrome is another rare inherited disease characterized by chromosomal instability syndromes such as aplastic anemia, leukemia, and breast or ovarian cancers [30]. Thus, genes associated with FA are also involved in DNA repair, especially DNA interstrand crosslink repair, and therefore mutations of these genes have the potential to impair the reprogramming process. To develop an FA-iPSC model, some groups have attempted to generate iPSCs from patients with FA who harbor FANCA or FANCD2, or from an FA mouse model with FANCA, FANCC, or FANCD1/BRCA2 (breast and ovarian cancer susceptibility protein 2$)[13,31,32]$. Although the FA-related genes do not induce early developmental lethality, iPSCs cannot be successfully generated until these genes are complementary to patient-derived fibroblasts [14]. Moreover, when mouse iPSCs were generated from FA mouse fibroblasts, the reprogramming efficiency was also found to be reduced or impaired $[13,20,32]$. BRCA1 coopts several FA proteins, including BRCA2 $[33,34]$. The complex formed by these proteins results in BRCA1 manifesting a similar phenotype to BRCA2, indicating that reprogramming from BRCA1-deficient mouse embryonic fibroblasts (MEFs) is also impaired in a similar manner to that observed in BRCA2deficient MEFs [20]. Meanwhile, as one of the interaction proteins of BRCAs, RAD51 plays a key role in the DNA repair process through homologous recombination. Similar to the low reprogramming efficiency induced by the functional loss of $B R C A$ genes, the silencing of RAD51 gene expression also seriously reduced reprogramming efficiency [20].

Besides a DNA repair system involving ATM-, FA-, BRCA-, or RAD51-dependent homologous recombination (HR), nonhomologous end joining (NHEJ) is also an important DNA repair system involving direct ligation of the end of the DNA strand break region. In this pathway, DNA ligase IV (encoded by the LIG4 gene) participates in repairing double-stranded breaks during the final step of NHEJ and $\mathrm{V}(\mathrm{D}) \mathrm{J}$ recombination $[35,36]$. Mutations in the LIG4 gene are associated with LIG4 syndrome, which is characterized by leukemia, immunodeficiency, and developmental retardation. In addition, although the consequences of this hypomorphic mutation of the LIG4 gene are less severe than the mouse embryonic lethality caused by the disrupted LIG4 gene, the reprogramming efficiency in cells derived from patients carrying the LIG4 gene mutation is significantly lower than that of normal control cells [15].

The examples highlighted above demonstrate that several chromosomal instability-related syndromes are associated with limitations in reprogramming, implying a strong link between reprogramming efficiency and genome instability (Table 1).

\subsection{The Constitutively Activated Bone Morphogenetic Pro-} tein (BMP) Signaling Pathway Affects Reprogramming. As members of transforming growth factor-beta, BMPs bind to BMP type II receptor, and then BMP-bound type II receptor kinase phosphorylates BMP type I receptor to activate Smad 
TABLE 1: Diseases with the inefficient reprogramming or inhibitory pluripotency maintenance.

\begin{tabular}{|c|c|c|c|c|c|}
\hline Category & Disease & Gene & Gene status & $\begin{array}{l}\text { Reprogramming efficiency } \\
\text { or } \\
\text { pluripotency maintenance }\end{array}$ & Refs. \\
\hline \multirow{5}{*}{$\begin{array}{l}\text { DNA } \\
\text { repair }\end{array}$} & $\begin{array}{c}\text { Ataxia } \\
\text { telangiectasia } \\
(\mathrm{A}-\mathrm{T})\end{array}$ & ATM & $\begin{array}{l}\text { 7004delCA, } \\
\text { 7886delTATTA }\end{array}$ & $\begin{array}{l}\text { Low efficient generation } \\
\qquad(15 \% \text { in } \\
\text { heterozygote)/extremely } \\
\text { low efficient generation } \\
\text { (4\% in homozygote) }\end{array}$ & {$[2]$} \\
\hline & $\begin{array}{l}\text { Fanconi anemia } \\
\text { (FA) }\end{array}$ & $\begin{array}{l}\text { FANCA, } \\
\text { FANCD2 }\end{array}$ & $\operatorname{del}^{\mathrm{a}}$ & $\begin{array}{l}\text { No reprogramming, or } \\
\text { extremely low efficient } \\
\text { generation (FANCA), low } \\
\text { efficient reprogramming } \\
\text { and poor maintenance } \\
\text { (FANCD2) }\end{array}$ & {$[13,14]$} \\
\hline & $\begin{array}{l}\text { FA or breast } \\
\text { cancer }\end{array}$ & BRCA1, BRCA2 & $\begin{array}{l}\text { ins }^{\mathrm{a}} \text { in exon } 11 \text { or } \mathrm{S} 1598 \mathrm{~F} \\
\text { point mutation for } \\
\text { BRCA1, del }{ }^{\mathrm{a}} \text { in exon } 27 \\
\text { for BRCA2 }\end{array}$ & $\sim 20$-fold lower than normal & {$[20]$} \\
\hline & Cancer & Rad51 & $\begin{array}{c}\text { Knockdown expression } \\
\text { by shRNA }\end{array}$ & $\sim 60$-fold lower than normal & {$[20]$} \\
\hline & LIG4 syndrome & LIG4 & $\begin{array}{c}\text { c. } 2440 \mathrm{C}>\mathrm{T} \text { in allele } 1 \\
\text { and c. } 1406 \mathrm{G}>\mathrm{A} \text { in allele } \\
2, \text { or } \mathrm{c} .833 \mathrm{G}>\mathrm{A}\end{array}$ & $\begin{array}{l}\text { Low reprogramming } \\
\text { efficiency }(0.002 \sim 0.012 \%) \\
\text { and apoptosis sensitivity }\end{array}$ & [15] \\
\hline Signaling & $\begin{array}{l}\text { Fibrodysplasia } \\
\text { ossificans } \\
\text { progressiva } \\
\text { (FOP) }\end{array}$ & ACVR1 & c. $617 \mathrm{G}>\mathrm{A}$ & $\begin{array}{l}\text { Low reprogramming } \\
\text { efficiency }(\sim 0.05 \%) \text { and } \\
\text { inhibitory maintenance of } \\
\text { iPSCs }\end{array}$ & {$[16,17]$} \\
\hline
\end{tabular}

${ }^{\mathrm{a}}$ Unknown locus.

proteins, including Smad 1/5/8, which play major roles in bone formation [37]. In addition to their roles in cellular differentiation, BMPs also regulate the histone $\mathrm{H} 3$ lysine 9 methylation that functionally impedes somatic cell reprogramming [38]. Thus, activation of the BMP signaling pathway prevents reprogramming beyond the intermediate preiPSCs stage [38]. Indeed, fibroblasts derived from patients with FOP syndrome that harbor an intrinsic abnormal BMP type I receptor also exhibit atypical reprogramming as well as inhibited self-renewal following incomplete reprogramming $[16,17]$. FOP is directly caused by mutations of the activin A receptor, type 1 ( $A C V R 1$ ) gene, which result in the synthesis of an abnormal activin receptor-like kinase 2 (ALK2) protein, leading to constitutive activity of BMP type I receptor and aberrant heterotopic ossification [37]. ALK2 has been shown to beneficially contribute to the efficacy of iPSCs generation only in the early phase of the reprogramming process, whereas constitutive ALK2 activation hampers iPSCs generation after the early phase [16]. In addition to the low reprogramming efficiency, mutant ALK2-iPSCs (mALK2iPSCs) phenotypically exhibit weak alkaline phosphatase activity, indicating incomplete reprogramming, as well as a tendency to differentiate into osteoblasts and mineralize, with high expression of representative osteogenic marker genes [17]. Therefore, mALK2-iPSCs cannot be efficiently stabilized or maintained unless the abnormal ALK2 protein is genetically treated or functionally weakened using ALK2 inhibitors.

\section{Gene-Correction Tools and Limitations of iPSC-Based Gene Therapy}

There are still many kinds of diseases that are not confirmed in reprogramming efficiency. And some of them would face the limitation of iPSC-based gene therapy due to the inefficient reprogramming affected by gene defect. However, as highlighted with the examples in the preceding sections, to overcome these limitations, it is necessary to completely remove the causal gene from the patient-derived somatic cells prior to the generation of iPSCs, or during the reprogramming process stage. Viral vector-mediated $F A$ gene therapy is the first example to overcome the limitation of iPSC-based gene therapy. Owing to the poor reprogramming ability of cells derived from patients with several FA-related disorders, treatment strategies have been initiated using recombinant viral vector-mediated gene therapy, thereby enabling the functional restoration of nonfunctional FA proteins. It is crucial to deliver the exogenous FA gene prior to reprogramming, as the nonfunctional FA gene impairs the reprogramming of patient-derived fibroblasts $[13,14]$. However, there are several hurdles to overcome the possibility of silence of the expression of normal gene delivered by viral vector, as well as simplifying the procedure to avoid the requirement of the numerous cumbersome steps involved, such as sorting out viral-genome-integrated cells, and then subsequently reprogramming based on the sorted cells. And although loss of a functional FA gene can be simply 


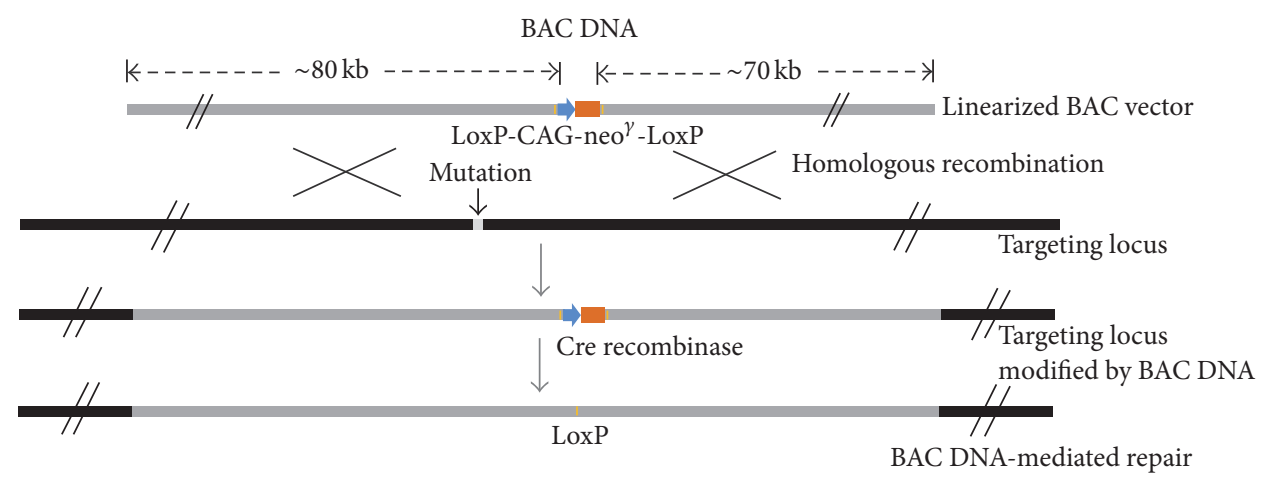

(a)

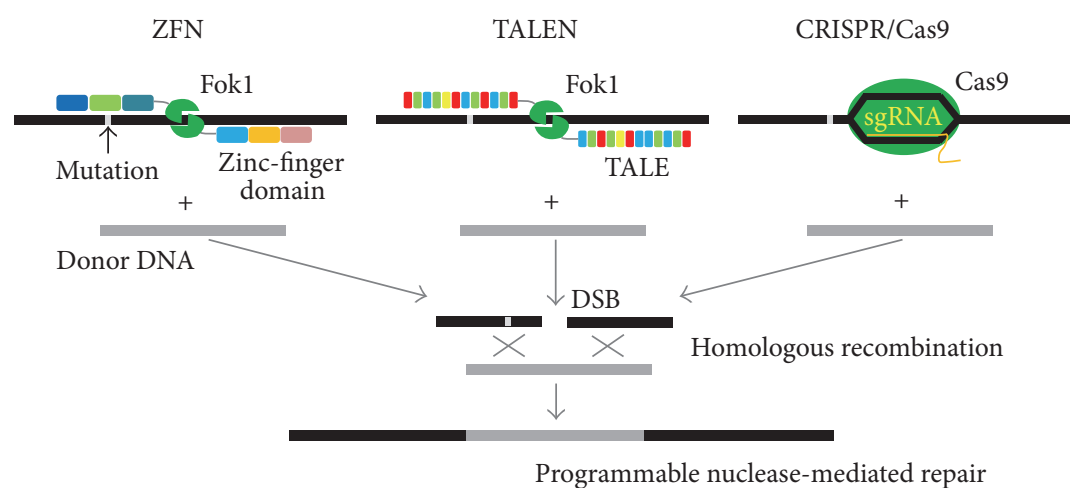

(b)

FIGURE 1: Mode of gene correction by gene-editing tools. (a) The image is drawn on the basis of the previous report [19]. The selection cassette $\left(\mathrm{CAG}-\mathrm{Neo}^{\gamma}\right)$ is inserted into the noncoding regions of the normal BAC DNA by recombineering. The lengths of flanking homology arms are indicated. The gene harboring a mutation base is replaced with the normal BAC DNA by homologous recombination. The selection cassette is removed by Cre recombinase. (b) The programmable nuclease modes, such as ZFN, TALEN, or CRISPR/Cas9, induce DNA doublestrand break (DSB) at the target locus. The mode of gene correction of programmable nucleases repairs DSB with the donor DNA, which is additionally introduced.

complemented by a normal exogenous FA gene, the viral vector-mediated gene therapy cannot be generally applied to the gain of functional gene, such as $A C V R 1$ c.617G $>A$, which constitutively activates the Smads signaling pathway as a dominant effect. That is, in order to overcome the limitation of iPSC-based gene therapy as the dominant effect of this mutation, a fundamental treatment must be applied to replace the mutated base with the wild type, simply not complemented by the normal gene.

This can best be accomplished by combining the benefits of several precise genome-editing tools such as powerful molecular scissors, including zinc-finger nucleases (ZFNs) [39-41], transcription activator-like effector nucleases (TALENs) [42, 43], RNA-guided endonucleases from the microbial clustered regularly interspaced short palindromic repeatCas9 (CRISPR-Cas9) nuclease systems [44-46], or bacterial artificial chromosome- (BAC-) based HR [19], enabling the target genes to be effectively inserted, deleted, or replaced in the genome as needed [17]. To date, the advantages and disadvantages of the ZFN, TALEN, and CRISPR/Cas9 systems have been comprehensively assessed [47]. However, the BAC-based HR system is not popular, despite showing high targeting efficiency for gene correction. The mode of BAC-based HR is by direct recombination of its own huge homologous arm, approximately $70-80 \mathrm{~kb}$ in length, at the targeting locus [19, 48, 49]; thus, this method does not require additional donor DNA (Figure 1(a)). By contrast, because the programmable nuclease modes, including $\mathrm{ZFN}$, TALEN, and CRISPR/Cas9, merely induce DNA doublestranded breaks at the target sequence, donor templates are additionally required to effectively mediate gene correction with homology-directed repair (Figure 1(b)).

To effectively apply gene-editing tools to iPSCs, the cells must be resistant to the stressful conditions required in the processes, including trypsinization or transfection. In general, the ROCK inhibitor Y-27632 has been shown to enhance the survivability of single cells dissociated by trypsin, helping to maintain the self-renewal capacity of the cells [50]. Nevertheless, Y-27632 treatment could not prevent mALK2-iPSCs from spontaneously differentiating under trypsinization and transfection, despite enhancing the viability of the cells [17]. Thus, we will suggest the challenges facing iPSC-based therapy approach in the next section. 


\section{Strategy to Overcome the Limitations of Stem Cell-Based Gene Therapy}

4.1. Combined Reprogramming and Gene Correction. The currently used methods for iPSC-based gene therapy generally involve the application of gene-correction tools in iPSCs after the reprogramming of donor-derived somatic cells (Figure 2(a); Step 1a to Step 2a). However, such methods rely on the assumption that the abnormal gene, which is involved in the disease, has no influence on the generation or maintenance of iPSCs (Figure 2(a); Step 1a). That is, if it were not possible to efficiently generate or maintain iPSCs derived from cells of a patient with one of the aforementioned diseases (Figure 2(a), Step 1b), the current gene therapy method would not be efficiently applicable to the pathogenic iPSCs. Therefore, to overcome this blind spot of conventional gene therapy, the abnormal genes would be first replaced with the normal gene in patient-derived somatic cells, prior to the iPSC stage. This can be accomplished by simultaneously introducing gene-correction tools along with reprogramming factors in the patient-derived somatic cells (Figure 2(b)) [17]. We applied this approach to mALK2 dermal fibroblasts, which exhibit atypical reprogramming and inhibit the maintenance of iPSCs [16, 17]. The expression of reprogramming factors coupled with gene-correction tools, including reprogramming episomal vectors, CRISPR/Cas9encoding vectors targeting the $A C V R 1$ gene, and donor DNA carrying the normal base, enabled the generation of normal ALK2-iPSCs without concerns regarding atypical reprogramming or maintenance inhibition caused by the gene mutation [17]. In addition to the application of this approach for vulnerable iPSCs, the one-step generation of gene-corrected iPSCs by simultaneous reprogramming and gene editing has additional advantages of saving time, effort, and cost $[17,51]$, thereby eliminating the need of performing the cumbersome reprogramming process and then subsequently performing gene editing separately, as required by the conventional gene-correction approach. Therefore, the one-step method described herein is highly practical and extensively applicable to cell-replacement therapies.

\subsection{Coupled TD and Gene Correction in LRRK2 (G2019S)} Somatic Cells. Familial PD caused by genetic mutation is generally rare, but one of the most frequent causes of earlyor late-onset $\mathrm{PD}$ results from an autosomal dominant mutant of LRRK2 (G2019S), which accounts for 5-6\% of all cases of familial PD or $1-2 \%$ of sporadic PD cases [52, 53]. And several groups have already demonstrated the ability to genetically correct LRRK2 (G2019S) mutant iPSCs using ZFN-mediated homology directed repair (HDR) [54, 55]. The ZFN-mediated HDR approach could be also performed using LRRK2 (G2019S) somatic cells by combining the reprogramming and gene-correction processes (Figure 3(a), Step 1b). Although this mutation has no effect on the reprogramming process or iPSC maintenance, it can nevertheless help to generate therapeutic iPSCs more rapidly than possible with a two-step generation method, involving reprogramming and subsequent gene correction (Figure 3(a), Step 1a to Step 2). Another attractive property of this approach is that these gene-editing tools can also be applied to iNSCs carrying LRRK2 (G2019S), because one of the main advantages of TD is the lack of risk of teratoma formation, which is a relevant concern with the use of iPSC-derived cells [56], and can rapidly generate lineage-specific cell types. However, the LRRK2 (G2019S) mutation can also cause the progressive degeneration of iNSCs, in which the iNSCs derived from mLRRK2-iPSCs exhibit depletion after undergoing several passages [18]. These findings strongly suggest that mLRRK2 exerts a negative influence on the maintenance of iNSCs derived from mLRRK2-somatic cells. Therefore, to directly generate iNSCs from PD fibroblasts with the LRRK2 (G2019S) mutation which are stably maintained, it is ideal to genetically treat the mLRRK2 fibroblasts during the process of TD, which may be achieved using a coupled TD and genecorrection approach (Figure 3(b), Step 1b). In contrast to the mLRRK2-iNSCs, the gene-corrected iNSCs would be stably self-renewing and differentiate toward dopaminergic neurons (DNs). The main critical factors of gene-corrected stem cells for application in cell-replacement therapy are the ability of continual growth, maintenance of their differentiation potential, and self-renewal ability. Several groups recently introduced methods for the direct conversion of human fibroblasts to DNs $[57,58]$. Thus, it could be conceptually possible to generate gene-corrected DNs that are induced directly from PD fibroblasts by coupling gene correction and direct conversion into DNs. However, the induced DNs have a general limitation of continuous growth, which hampers the ability to screen for positive clones as well as to acquire a sufficient amount of therapeutic material. Thus, along with the concurrent reprogramming and gene-correction approach, the application of combined gene correction and TD-mediated iNSC appears to be a suitable approach to generate the therapeutic materials for PD cell therapy.

\section{Conclusions}

To date, numerous types of patient-derived iPSCs have been generated, most of which have been provided as resources for gene therapy. However, there are several diseases for which treatment using current iPSCs-based gene therapy approaches remains a challenge.

Several of these diseases result from chromosomal instability or impaired DNA repair. Although even the generation of low numbers of disease-derived iPSCs is still promising, cases with a high incidence of aneuploidy can be further exacerbated by the reprogramming factors such as OCT4, SOX2, KLF4, and c-MYC. Indeed, ATM-deficient iPSCs acquired serious chromosomal abnormalities after several passages [29]. Although c-MYC, a representative oncoprotein, enhances reprogramming efficiency, it may also substantially increase the probability of abnormalities in reprogrammed cells with a defective DNA repair gene. However, as the proteins involved in DNA repair are normally rescued with the use of the one-step system proposed herein, the reprogramming efficiency could be enhanced, thereby reducing the incidence of aneuploidy.

As the one-step system has been recently introduced, only four cases applying this approach have been reported to date 


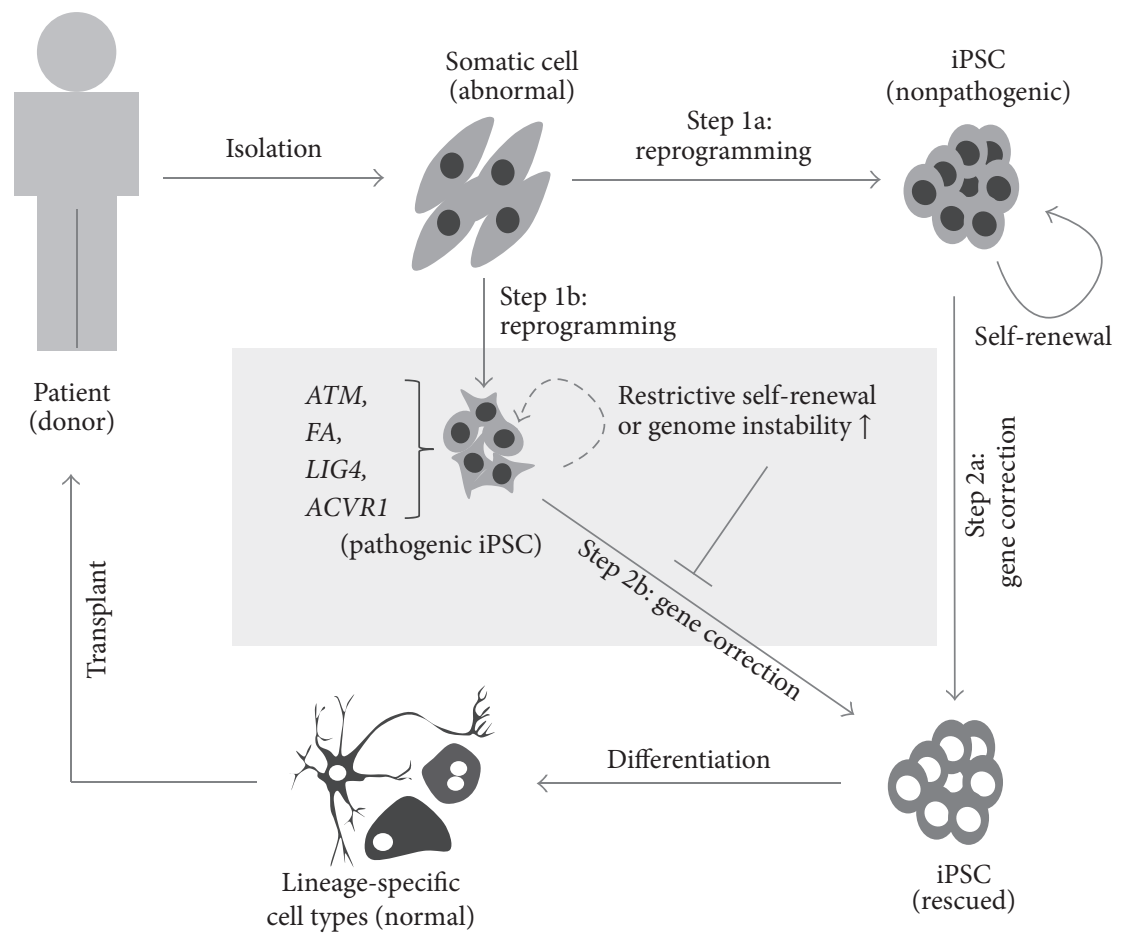

(a)
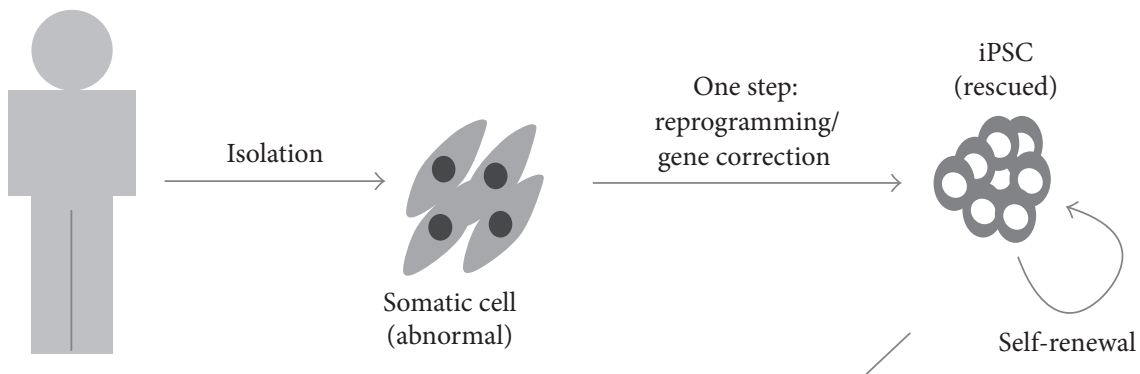

Patient
(donor)

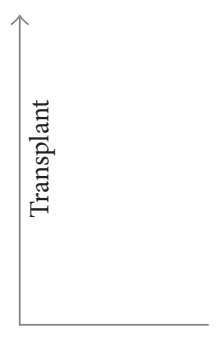

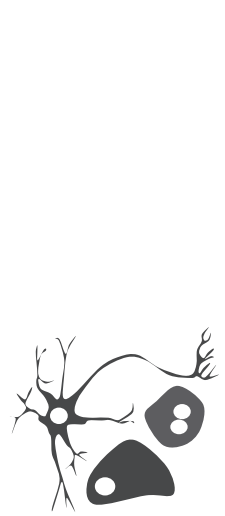

Lineage-specific cell types (normal)

(b)

FIGURE 2: Overview of the combined reprogramming and gene-correction strategy. (a) Current iPSC-based gene-correction approach. Step 1a: nonpathogenic iPSCs can stably maintain their self-renewal property. Step 1b: pathogenic iPSCs, derived from cells with mutations in ATM, FA, LIG4, or ACVR1, exhibit restrictive self-renewal and a potential rise of genome instability, hampering progress to Step 2b. Step 2a: the iPSCs generated from Step 1a can be subsequently subject to gene correction with various tools such as ZFNs, TALENs, CRISPR/Cas9, or BAC vectors. (b) A one-step process involving simultaneous reprogramming and gene correction. Gene-corrected iPSCs are directly produced from patient-derived somatic cells, concurrently combining the reprogramming factors and gene-correction tools. 


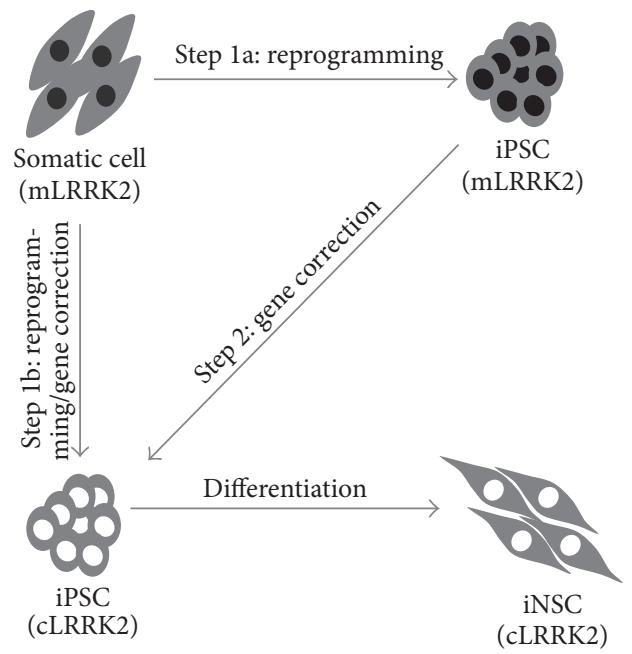

(a)

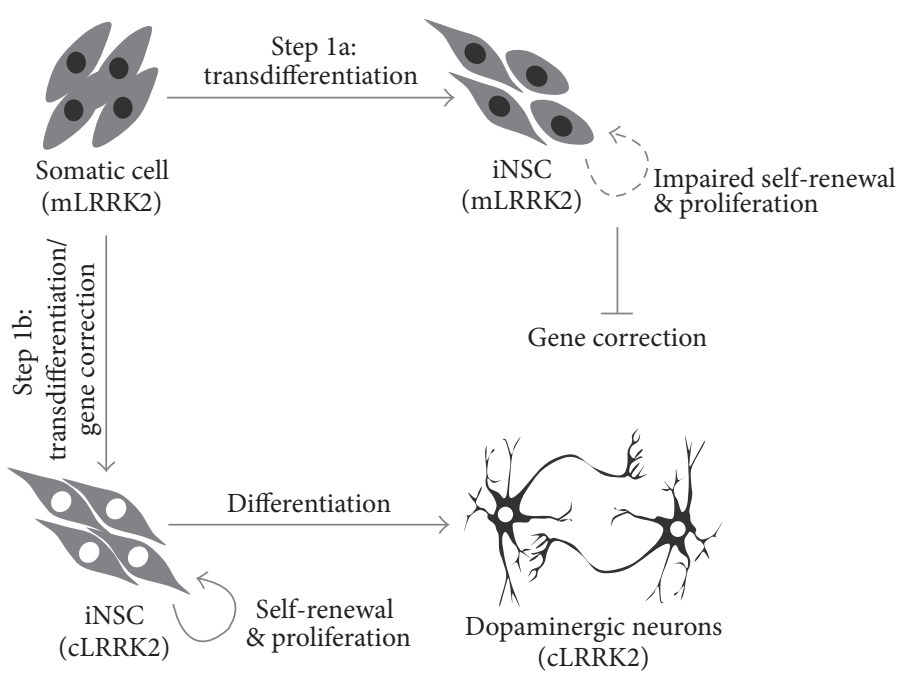

(b)

FIGURE 3: Application of the gene-correction approach in cells with the LRRK2 (G2019S) mutation. (a) Conventional gene-correction approach using mutant iPSCs generated from LRRK2 (G2019S) mutant fibroblasts, involving reprogramming with subsequent gene correction (Step 2 via Step 1a), and the combined reprogramming and gene-correction approach based on LRRK2 (G2019S) mutant fibroblasts (Step 1b). (b) Mutant iNSCs are generated from LRRK2 (G2019S) mutant fibroblasts, potentially exhibiting impaired self-renewal or proliferation in Step 1a, whereas, in Step 1b, transdifferentiation and gene correction occur simultaneously and the corrected iNSCs are capable of stable proliferation with maintained self-renewal potential.

$[17,51,59]$. In all cases, the cells were genetically treated with CRISPR/Cas9-mediated HDR, resulting in a targeting efficiency of $5-17 \%[17,51,59]$. This targeting efficiency is comparable to that obtained with iPSC-based approaches, which has been attempted using the diverse gene therapy tools available, such as plasmid, BAC, adeno-associated virus, helper-dependent adenovirus, and ZFN [60].

LRRK2 (G2019S) interacts with lamin B1 protein, which is anchored to the inner nuclear membrane and is involved in breaking the framework of the nuclear envelope, leading to the loss of function of lamin B1 protein, which ultimately increases chromatin instability and eventually depletes the iNSC pool. Therefore, the use of TD-mediated iNSCs coupled with gene correction may enable the maintenance of the chromatin stability of iNSCs, thereby providing a stable therapeutic method for cell replacement in patients with PD.

In addition to gene therapy for the rare diseases mentioned above, the advanced one-step method is generally applicable to all disease-derived cells regardless of the defective gene involved, which can substantially save time, reduce the need for labor-intensive experiments, and cut the cost required for current iPSC-based gene therapy methods by half. Therefore, the one-step method described herein is expected to become increasingly popular for the development of more rapid and personalized cell-replacement therapies in the near future.

\section{Competing Interests}

The authors declare that there are no competing interests regarding the publication of this paper.

\section{Acknowledgments}

This work was supported in part by the National Research Foundation of Korea Grants 2013M3A9B4076487 and 2014M3A9D7034335 and the Korea Institute of Oriental Medicine Grants K16091 and K16130.

\section{References}

[1] S. Yamanaka, "Induced pluripotent stem cells: past, present, and future," Cell Stem Cell, vol. 10, no. 6, pp. 678-684, 2012.

[2] G. Lee and L. Studer, "Induced pluripotent stem cell technology for the study of human disease," Nature Methods, vol. 7, no. 1, pp. 25-27, 2010.

[3] I.-H. Park, N. Arora, H. Huo et al., "Disease-specific induced pluripotent stem cells," Cell, vol. 134, no. 5, pp. 877-886, 2008.

[4] J. T. Dimos, K. T. Rodolfa, K. K. Niakan et al., "Induced pluripotent stem cells generated from patients with ALS can be differentiated into motor neurons," Science, vol. 321, no. 5893, pp. 1218-1221, 2008.

[5] G. Lee, E. P. Papapetrou, H. Kim et al., "Modelling pathogenesis and treatment of familial dysautonomia using patient-specific iPSCs," Nature, vol. 461, no. 7262, pp. 402-406, 2009.

[6] X. Carvajal-Vergara, A. Sevilla, S. L. Dsouza et al., "Patientspecific induced pluripotent stem-cell-derived models of LEOPARD syndrome," Nature, vol. 465, no. 7299, pp. 808-812, 2010.

[7] J. Zhang, Q. Lian, G. Zhu et al., "A human iPSC model of hutchinson gilford progeria reveals vascular smooth muscle and mesenchymal stem cell defects," Cell Stem Cell, vol. 8, no. 1, pp. 31-45, 2011. 
[8] M. C. N. Marchetto, C. Carromeu, A. Acab et al., "A model for neural development and treatment of rett syndrome using human induced pluripotent stem cells," Cell, vol. 143, no. 4, pp. 527-539, 2010.

[9] A. D. Ebert, J. Yu, F. F. Rose Jr. et al., "Induced pluripotent stem cells from a spinal muscular atrophy patient," Nature, vol. 457, no. 7227, pp. 277-280, 2009.

[10] S. Agarwal, Y.-H. Loh, E. M. McLoughlin et al., "Telomere elongation in induced pluripotent stem cells from dyskeratosis congenita patients," Nature, vol. 464, no. 7286, pp. 292-296, 2010.

[11] M. Yazawa, B. Hsueh, X. Jia et al., "Using induced pluripotent stem cells to investigate cardiac phenotypes in Timothy syndrome," Nature, vol. 471, no. 7337, pp. 230-236, 2011.

[12] S. Nayler, M. Gatei, S. Kozlov et al., "Induced pluripotent stem cells from ataxia-telangiectasia recapitulate the cellular phenotype," Stem Cells Translational Medicine, vol. 1, no. 7, pp. 523-535, 2012.

[13] L. U. W. Müller, M. D. Milsom, C. E. Harris et al., "Overcoming reprogramming resistance of Fanconi anemia cells," Blood, vol. 119, no. 23, pp. 5449-5457, 2012.

[14] Á. Raya, I. Rodríguez-Piz, G. Guenechea et al., "Diseasecorrected haematopoietic progenitors from Fanconi anaemia induced pluripotent stem cells," Nature, vol. 460, no. 7251, pp. 53-59, 2009.

[15] K. Tilgner, I. Neganova, I. Moreno-Gimeno et al., "A human iPSC model of Ligase IV deficiency reveals an important role for NHEJ-mediated-DSB repair in the survival and genomic stability of induced pluripotent stem cells and emerging haematopoietic progenitors," Cell Death and Differentiation, vol. 20, no. 8, pp. 1089-1100, 2013.

[16] M. Hamasaki, Y. Hashizume, Y. Yamada et al., "Pathogenic mutation of ALK2 inhibits induced pluripotent stem cell reprogramming and maintenance: mechanisms of reprogramming and strategy for drug identification," Stem Cells, vol. 30, no. 11, pp. 2437-2449, 2012.

[17] B. Y. Kim, S. Jeong, S. Y. Lee et al., "Concurrent progress of reprogramming and gene correction to overcome therapeutic limitation of mutant ALK2-iPSC," Experimental \& Molecular Medicine, vol. 48, article e237, 2016.

[18] G.-H. Liu, J. Qu, K. Suzuki et al., "Progressive degeneration of human neural stem cells caused by pathogenic LRRK2," Nature, vol. 491, no. 7425, pp. 603-607, 2012.

[19] H. Song, S.-K. Chung, and Y. Xu, "Modeling disease in human ESCs using an efficient BAC-based homologous recombination system," Cell Stem Cell, vol. 6, no. 1, pp. 80-89, 2010.

[20] F. González, D. Georgieva, F. Vanoli et al., "Homologous recombination DNA repair genes play a critical role in reprogramming to a pluripotent state," Cell Reports, vol. 3, no. 3, pp. 651-660, 2013.

[21] B. B. Lake, J. Fink, L. Klemetsaune et al., "Context-dependent enhancement of induced pluripotent stem cell reprogramming by silencing Puma," STEM CELLS, vol. 30, no. 5, pp. 888-897, 2012.

[22] Y. Li, H. Feng, H. Gu et al., "The p53-PUMA axis suppresses iPSC generation," Nature Communications, vol. 4, article 2174, 2013.

[23] R. M. Marión, K. Strati, H. Li et al., "A p53-mediated DNA damage response limits reprogramming to ensure iPS cell genomic integrity," Nature, vol. 460, no. 7259, pp. 1149-1153, 2009.
[24] H. Hong, K. Takahashi, T. Ichisaka et al., "Suppression of induced pluripotent stem cell generation by the p53-p21 pathway," Nature, vol. 460, no. 7259, pp. 1132-1135, 2009.

[25] H. Li, M. Collado, A. Villasante et al., "The Ink4/Arf locus is a barrier for iPS cell reprogramming," Nature, vol. 460, no. 7259, pp. 1136-1139, 2009.

[26] A. Banito, S. T. Rashid, J. C. Acosta et al., "Senescence impairs successful reprogramming to pluripotent stem cells," Genes and Development, vol. 23, no. 18, pp. 2134-2139, 2009.

[27] M. A. Rasmussen, B. Holst, Z. Tümer et al., “Transient p53 suppression increases reprogramming of human fibroblasts without affecting apoptosis and DNA damage," Stem Cell Reports, vol. 3, no. 3, pp. 404-413, 2014.

[28] G. Rotman and Y. Shiloh, "ATM: from gene to function," Human Molecular Genetics, vol. 7, no. 10, pp. 1555-1563, 1998.

[29] T. Kinoshita, G. Nagamatsu, T. Kosaka et al., "Ataxiatelangiectasia mutated (ATM) deficiency decreases reprogramming efficiency and leads to genomic instability in iPS cells," Biochemical and Biophysical Research Communications, vol. 407, no. 2, pp. 321-326, 2011.

[30] M. D. Tischkowitz and S. V. Hodgson, "Fanconi anaemia," Journal of Medical Genetics, vol. 40, no. 1, pp. 1-10, 2003.

[31] G.-H. Liu, K. Suzuki, M. Li et al., "Modelling Fanconi anemia pathogenesis and therapeutics using integration-free patientderived iPSCs," Nature Communications, vol. 5, article 4330, 2014.

[32] S. Navarro, V. Moleiro, F. J. Molina-Estevez et al., "Generation of iPSCs from genetically corrected Brca2 hypomorphic cells: implications in cell reprogramming and stem cell therapy," STEM CELLS, vol. 32, no. 2, pp. 436-446, 2014.

[33] F. Zhang, J. Ma, J. Wu et al., "PALB2 Links BRCA1 and BRCA2 in the DNA-damage response," Current Biology, vol. 19, no. 6, pp. 524-529, 2009.

[34] T. Taniguchi, I. Garcia-Higuera, P. R. Andreassen, R. C. Gregory, M. Grompe, and A. D. D'Andrea, "S-phase-specific interaction of the Fanconi anemia protein, FANCD2, with BRCA1 and RAD51," Blood, vol. 100, no. 7, pp. 2414-2420, 2002.

[35] N. E. Sharpless, D. O. Ferguson, R. C. O’Hagan et al., "Impaired nonhomologous end-joining provokes soft tissue sarcomas harboring chromosomal translocations, amplifications, and deletions," Molecular Cell, vol. 8, no. 6, pp. 1187-1196, 2001.

[36] M. O’Driscoll, K. M. Cerosaletti, P.-M. Girard et al., "DNA ligase IV mutations identified in patients exhibiting developmental delay and immunodeficiency," Molecular Cell, vol. 8, no. 6, pp. 1175-1185, 2001.

[37] T. Fukuda, M. Kohda, K. Kanomata et al., "Constitutively activated ALK2 and increased SMAD1/5 cooperatively induce bone morphogenetic protein signaling in fibrodysplasia ossificans progressiva," Journal of Biological Chemistry, vol. 284, no. 11, pp. 7149-7156, 2009.

[38] J. Chen, H. Liu, J. Liu et al., "H3K9 methylation is a barrier during somatic cell reprogramming into iPSCs," Nature Genetics, vol. 45, no. 1, pp. 34-42, 2013.

[39] Y.-G. Kim, J. Cha, and S. Chandrasegaran, "Hybrid restriction enzymes: zinc finger fusions to Fok I cleavage domain," Proceedings of the National Academy of Sciences of the United States of America, vol. 93, no. 3, pp. 1156-1160, 1996.

[40] M. Bibikova, K. Beumer, J. K. Trautman, and D. Carroll, "Enhancing gene targeting with designed zinc finger nucleases," Science, vol. 300, no. 5620, p. 764, 2003. 
[41] S. Ramalingam, V. London, K. Kandavelou et al., "Generation and genetic engineering of human induced pluripotent stem cells using designed zinc finger nucleases," Stem Cells and Development, vol. 22, no. 4, pp. 595-610, 2013.

[42] D. Hockemeyer, H. Wang, S. Kiani et al., "Genetic engineering of human pluripotent cells using TALE nucleases," Nature Biotechnology, vol. 29, no. 8, pp. 731-734, 2011.

[43] J. C. Miller, S. Tan, G. Qiao et al., "A TALE nuclease architecture for efficient genome editing," Nature Biotechnology, vol. 29, no. 2, pp. 143-150, 2011.

[44] S. W. Cho, S. Kim, J. M. Kim, and J.-S. Kim, "Targeted genome engineering in human cells with the Cas9 RNA-guided endonuclease," Nature Biotechnology, vol. 31, no. 3, pp. 230-232, 2013.

[45] L. Cong, F. A. Ran, D. Cox et al., "Multiplex genome engineering using CRISPR/Cas systems," Science, vol. 339, no. 6121, pp. 819823, 2013.

[46] P. Mali, L. Yang, K. M. Esvelt et al., "RNA-guided human genome engineering via Cas9," Science, vol. 339, no. 6121, pp. 823-826, 2013.

[47] T. Gaj, C. A. Gersbach, and C. F. Barbas, "ZFN, TALEN, and CRISPR/Cas-based methods for genome engineering," Trends in Biotechnology, vol. 31, no. 7, pp. 397-405, 2013.

[48] Z.-N. Zhang, S.-K. Chung, Z. Xu, and Y. Xu, "Oct4 maintains the pluripotency of human embryonic stem cells by inactivating p53 through sirtl-mediated deacetylation," Stem Cells, vol. 32, no. 1, pp. 157-165, 2014.

[49] S.-K. Chung, S. Zhu, Y. Xu, and X. Fu, "Functional analysis of the acetylation of human p53 in DNA damage responses," Protein and Cell, vol. 5, no. 7, pp. 544-551, 2014.

[50] K. Watanabe, M. Ueno, D. Kamiya et al., "A ROCK inhibitor permits survival of dissociated human embryonic stem cells," Nature Biotechnology, vol. 25, no. 6, pp. 681-686, 2007.

[51] S. E. Howden, J. P. Maufort, B. M. Duffin, A. G. Elefanty, E. G. Stanley, and J. A. Thomson, "Simultaneous reprogramming and gene correction of patient fibroblasts," Stem Cell Reports, vol. 5, no. 6, pp. 1109-1118, 2015.

[52] D. M. Kay, C. P. Zabetian, S. A. Factor et al., "Parkinson's disease and LRRK2: frequency of a common mutation in U.S. movement disorder clinics," Movement Disorders, vol. 21, no. 4, pp. 519-523, 2006.

[53] D. Berg, K. Schweitzer, P. Leitner et al., “Type and frequency of mutations in the LRRK2 gene in familial and sporadic Parkinson's disease," Brain, vol. 128, no. 12, pp. 3000-3011, 2005.

[54] F. Soldner, J. Laganiere, A. W. Cheng et al., "Generation of isogenic pluripotent stem cells differing exclusively at two early onset Parkinson point mutations," Cell, vol. 146, no. 2, pp. 318331, 2011.

[55] P. Reinhardt, B. Schmid, L. F. Burbulla et al., "Genetic correction of a lrrk2 mutation in human iPSCs links parkinsonian neurodegeneration to ERK-dependent changes in gene expression," Cell Stem Cell, vol. 12, no. 3, pp. 354-367, 2013.

[56] W. T. Wong and J. P. Cooke, "Therapeutic transdifferentiation of human fibroblasts into endothelial cells using forced expression of lineage-specific transcription factors," Journal of Tissue Engineering, vol. 7, pp. 1-10, 2016.

[57] U. Pfisterer, A. Kirkeby, O. Torper et al., "Direct conversion of human fibroblasts to dopaminergic neurons," Proceedings of the National Academy of Sciences of the United States of America, vol. 108, no. 25, pp. 10343-10348, 2011.
[58] X. Liu, F. Li, E. A. Stubblefield et al., "Direct reprogramming of human fibroblasts into dopaminergic neuron-like cells," Cell Research, vol. 22, no. 2, pp. 321-332, 2012.

[59] S. Howden, B. McColl, A. Glaser et al., "A Cas9 variant for efficient generation of indel-free knockin or gene-corrected human pluripotent stem cells," Stem Cell Reports, vol. 7, no. 3, pp. 508-517, 2016.

[60] I. Sancho-Martinez, M. Li, and J. C. Izpisua Belmonte, "Disease correction the iPSC way: advances in iPSC-based therapy," Clinical Pharmacology and Therapeutics, vol. 89, no. 5, pp. 746749, 2011. 

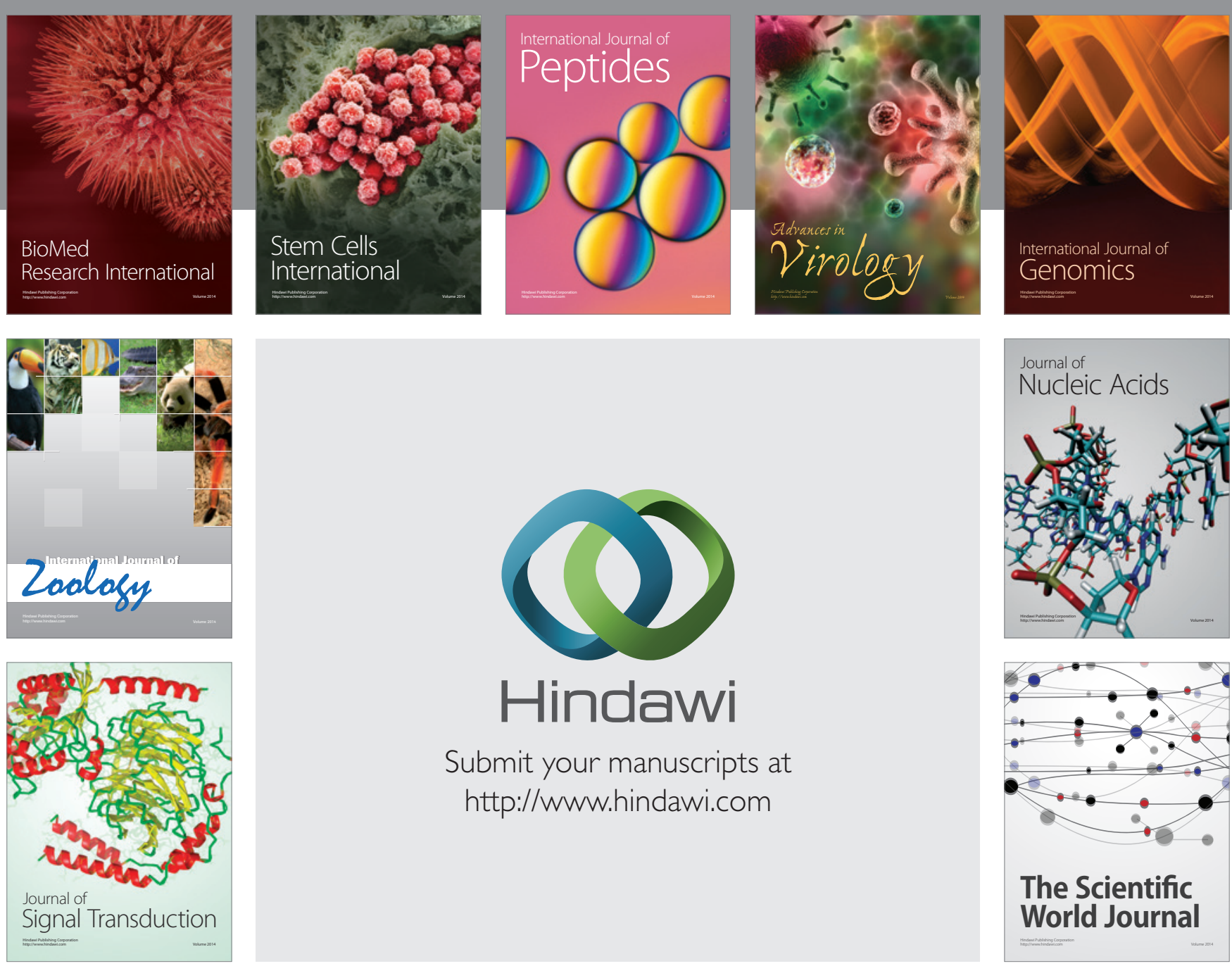

Submit your manuscripts at

http://www.hindawi.com
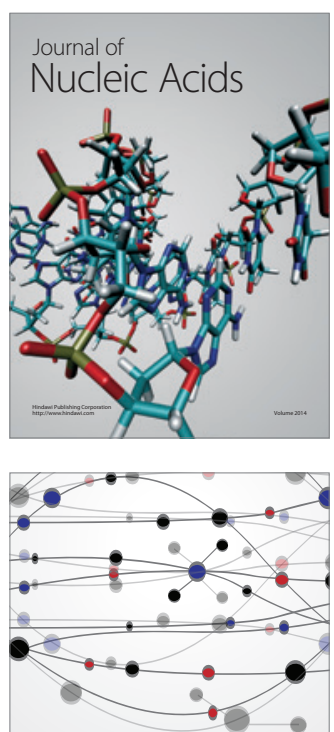

The Scientific World Journal
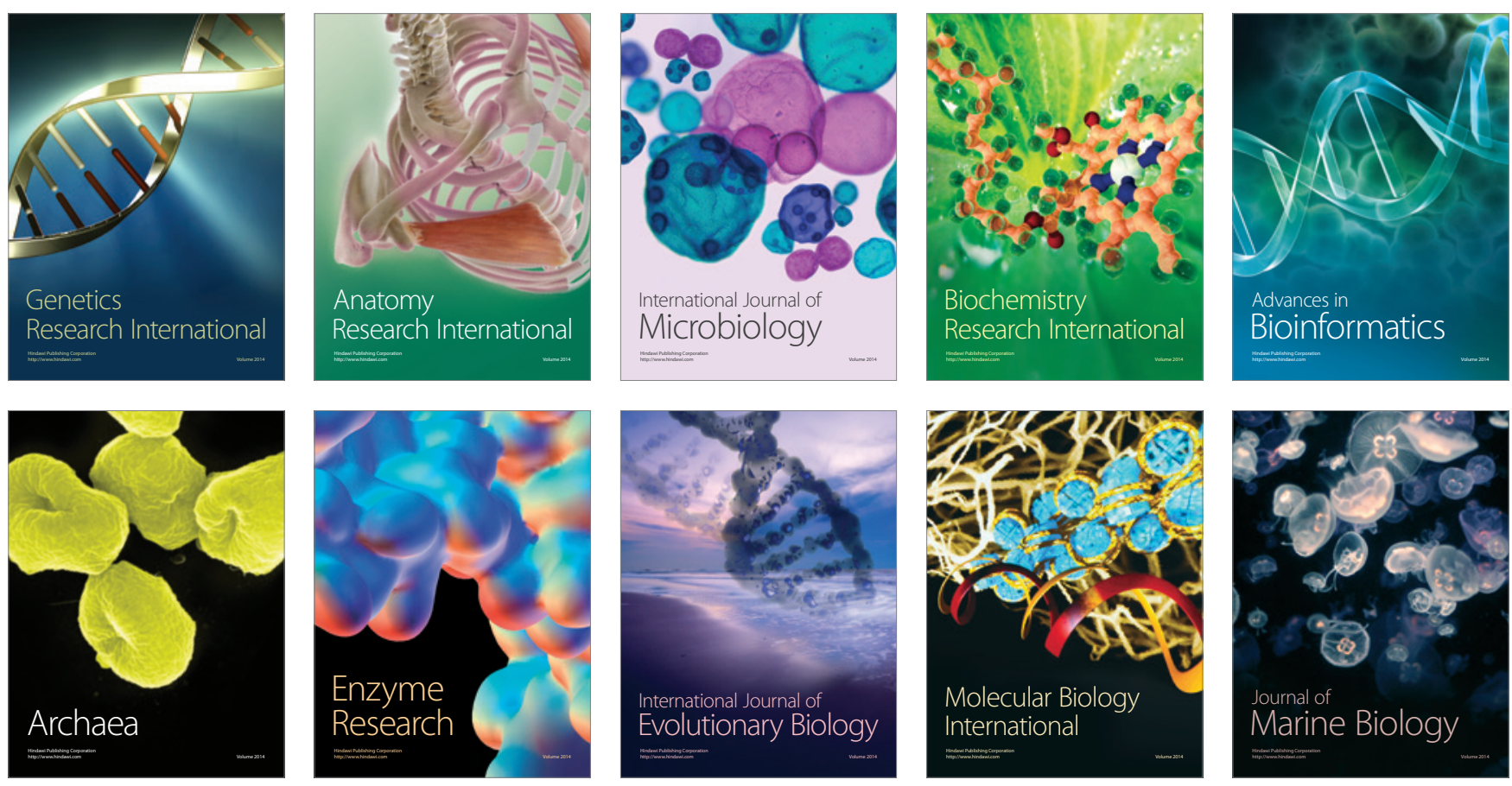